\title{
Rachitisme hypophosphatémique héréditaire
}

Le rachitisme hypophosphatémique lié au chromosome $\mathrm{X}$ de la souris $H y p$ constitue un intéressant modèle du rachitisme héréditaire chez l'homme.

\section{Harriet S. Tenenhouse Professeur adjoint, département de pédiatrie.}

\section{RÉFÉRENCES}

I. Rasmussen H, Anast C. Familial hypophosphatemic rickets and vitamin D-dependent rickets. In: Stanbury JB, Wyngaarden JB, Fredrickson DS, Goldstein JL, Brown MS, eds. The Metabolic Basis of Inherited Disease. New York: McGraw Hill Book Co., 1983: 1 743-73.

2. Eicher EM, Southard JL, Scriver CR, Glorieux $\mathrm{FH}$. Hypophosphatemia: Mouse model for human familial hypophosphatemic (vitamin D-resistant) rickets. Proc Natl Acad Sci USA 1976; 73: 4667-7I.

3. Cowgill LD, Goldfarb S, Lau K, Slatopolsky $\mathrm{E}$, Agus ZS. Evidence for an intrinsic renal tubular defect in mice with genetic hypophosphatemic rickets. 7 Clin Invest 1979; 63: 1203-10.

\section{ADRESSE}

H. S. Tenenhouse : Groupe de recherche génétique, institut de recherches, hôpital de Montréal pour les enf ants et département de biologie, centre de génétique humaine, université McGill, 2300 rue Tupper, $\mathrm{H}_{3} \mathrm{H}_{1} \mathrm{P}_{3}$, Montréal.

$m / s n^{\circ} 8$, vol. 2, octobre 86 réabsorber le phosphate filtré $[\mathrm{I}, 2]$. La concentration circulante d'hormone parathyroïdienne étant normale au cours de cette maladie chez l'homme comme chez la souris, et le défaut de réabsorption rénale du phosphore persistant après parathyroïdectomie [I, 3], il paraît clair que le phénotype observé n'est pas dû à un hyperparathyroïdisme. Ce tableau suggère donc que le défaut primaire dans cette maladie murine ou humaine, se trouve dans le rein. A cause des similarités phénotypiques entre la maladie affectant l'organisme humain et celui de la souris, et parce que les gènes présents sur le chromosome $X$ sont conservés durant l'évolution des mammifères, il est hautement probable que le gène mutant soit homologue dans les deux espèces. Pour cette raison, la souris hypophosphatémique constitue un modèle utile qui devrait permettre d'élucider les bases moléculaires et biochimiques du défaut induit par ce gène mutant.

On discutera dans cet article du phénotype rénal de la souris hypophosphatémique et l'on présentera plusieurs hypothèses pour expliquer comment la mutation d'un seul gène pourrait rendre 


\section{RÉFÉRENCES}

4. Giasson SD, Brunette MG, Danan G, Vigneault N, Carrière $S$. Micropuncture studiy of renal phosphorus transport in hypophosphatemic vitamin D-resistant rickets mice. Pfluegers Arch 1977; 37 I : 33-8.

5. Tenenhouse HS, Scriver CR, McInnes RR, Glorieux FH. Renal handling of phosphate in vivo and in vitro by the $\mathrm{X}$-linked hypophosphatemic male mouse: Evidence for a defect in the brush border membrane. Kidney Int 1978; 14: 236-44.

6. Tenenhouse HS, Cole DEC, Scriver CR Mendelian hypophosphatemias as probes of phosphate and sulphate transport by mammalian kidney. In: Belton NR, Toothill C, eds. Transport and Inherited Disease. Lancaster, Boston, La Hague: MTP Press Ltd, 1 98I: 231-62.

7. Tenenhouse HS, Scriver CR. Renal brush border membrane adaptation to phosphorus deprivation in the $H y p / \mathrm{Y}$ mouse. Nature 1979 281: 225-7.

8. Kessler RJ, Vaughn DA, Fannestil DD Phosphate-binding proteolipid from brush border. 7 Biol Chem 1982; 257: 1431 I-7.

9. Kessler RJ, Vaughn DA, Tenenhouse HS Recovery and phosphate binding activity of phosphorin is not reduced in renal brush border membranes of $H y p$ mice. 7 Bone and Mineral Res 1986; 1:105 (Abstract).

10. Tenenhouse HS, Fast DK, Scriver CR, Kol tay M. Intestinal transport of phosphate is not impaired in the Hyp (Hypophosphatemic) mouse Biochem Biophys Res Commun 1981; 100: 537-43.

II. Cunningham J, Gomes H, Seino Y, Chase LR. Abnormal 24-hydroxylation of 25-hydroxyvitamin $\mathrm{D}$ in the $\mathrm{X}$-linked hypophosphatemic mouse. Endocrinology 1983; 112 : 633-8.

12. Lobaugh B, Drezner MK. Abnormal regulation of renal 25-hydroxyvitamin D-I $\alpha$-hydroxylase activity in the $\mathrm{X}$-linked hypophosphatemic mouse. 7 Clin Invest 1983; 7 1: 400-3.

13. Tenenhouse HS. Abnormal renal mitochondrial 25-hydroxyvitamin $D_{3}-1$-hydroxylase activity in the vitamin $\mathrm{D}$ and calcium deficient X-linked Hyp mouse. Endocrinology 1983; I I3: 8I6-8.

14. Tenenhouse HS. Investigation of the mechanism for abnormal renal 25-hydroxyvitamin $\mathrm{D}_{3}$ I-hydroxylase activity in the X-linked $H y p$ mouse. Endocrinology 1984; 11 5:634-9.

I5. Fukase M, Avioli LV, Birge SJ, Chase LR Abnormal regulation of 25-hydroxyvitamin $\mathrm{D}_{3}-\mathrm{I} \alpha$-hydroxylase activity by calcium and calcitonin in renal cortex from hypophosphatemic
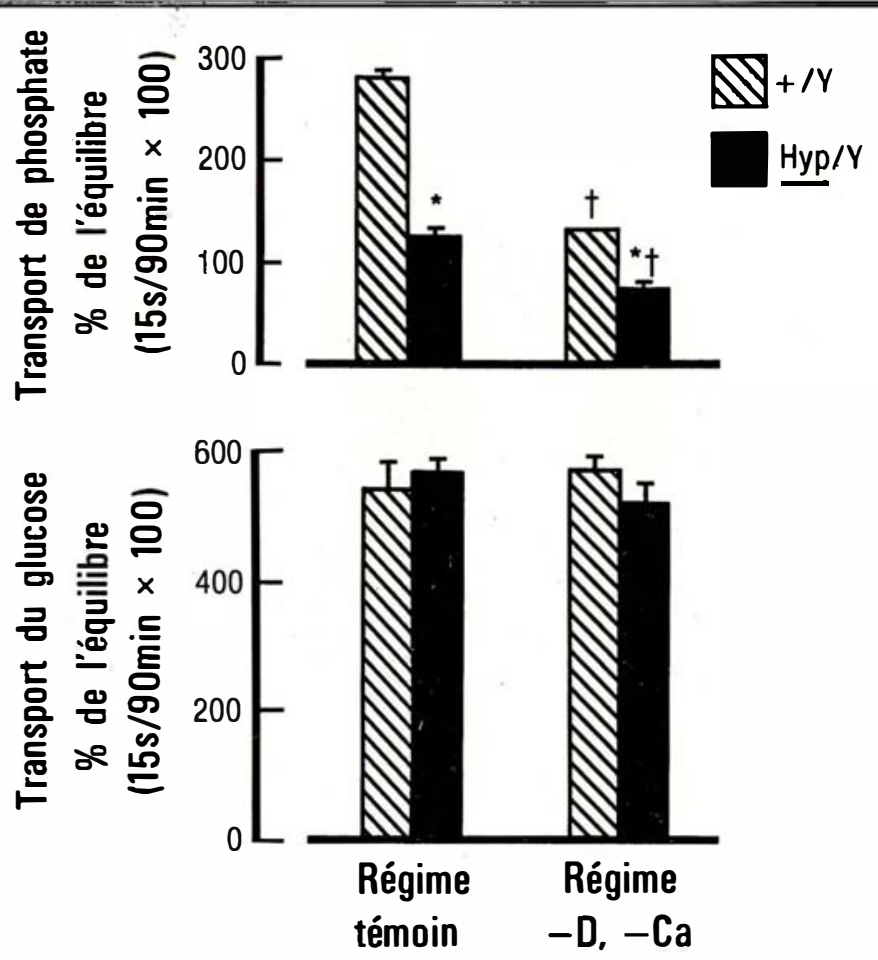

Figure 1. Effets combinés d'un régime carencé en calcium et en vitamine $D$ sur les co-transports sodium-phosphate et sodiumglucose par les bordures en brosse de souris mutantes. Les captations de phosphate $(0,1 \mathrm{mM})$ ou de glucose $(0,01 \mathrm{mM})$ sont mesurées à $15 \mathrm{sec}$ et 90 min. Chaque point représente la moyenne +1 écart-type de six déterminations obtenues de trois préparations différentes dé vésicules dans chaque groupe. * Effet du génotype; †effet du régime chez les souris normales par rapport aux souris mutantes $p<0,001$.

compte de la diversité des anomalies biochimiques caractéristiques de cette affection de la souris.

\section{Transport rénal des phosphates}

Comme chez l'homme porteur d'une HLX, l'excrétion urinaire de phosphate est anormalement haute par rapport à la phosphorémie chez la souris $H y p$ [2]. Les études de microponction de Giasson et collaborateurs ont localisé le défaut du transport du phosphore dans le tube proximal du rein de cette espèce [4]. Des études antérieures de notre laboratoire avaient démontré que la captation du phosphore par des tranches de cortex rénal était normale chez la souris Hyp [5]. Étant donné qu'avec cette préparation c'est surtout la surface basolatérale des cellules épithéliales rénales qui est exposée et qui transporte le phosphore, nos résultats suggéraient que le transport de phosphore par la membrane basolatérale était fonctionnellement normal dans ce modèle [5]. Au contraire, le transport de phosphate lié au sodium par des vésicules de bordure en brosse préparées à partir de reins de souris $H y p$ est abaissé à $50 \%$ de la normale [5] (figure I). Ce défaut de transport est spécifique pour le phosphate [5] ( figure I) et est dû à une chute de $50 \%$ de la Vmax* apparente de ce processus [6]. La Km** apparente de ce transport n'est pas différente chez les

\footnotetext{
*Vmax : constante cinétique signifiant * vitesse maximale ".

** Km : constante cinétique signifiant " constante de Michaelis", elle est une mesure de l'affinité d'une enzyme ou d'un système enzymatique pour un substrat.
} 
souris normales et les souris mutantes [6].

Nous avons également démontré que le fait de placer les souris normales ou hypophosphatémiques sous régime pauvre en phosphore augmente d'un facteur de deux le transport du phosphore par les vésicules de bordure en brosse rénales [7]. Ceci indique que les animaux porteurs des deux génotypes conservent la possibilité de s'adapter à un régime déficient en phosphore. Néanmoins, le transport de phosphore par les vésicules provenant de souris $H y p$ demeure à la moitié de la valeur observée chez la souris normale. Des études récentes ont démontré que la présence et les caractéristiques de liaison de la phosphorine, un protéolipide de faible poids moléculaire présent dans la membrane de la bordure en brosse et intervenant dans le transport du phosphore [8], sont normales chez la souris $H y p$ [9], ce qui suggère que le défaut en cause implique une autre sous-unité ou bien un régulateur de ce système de transport. Il est intéressant de noter que le transport du phosphore dépendant du sodium, par des anses intestinales éversées ou par des préparations purifiées de vésicules intestinales, demeure normal chez la souris $H y p$ [ı]. Ces résultats suggèrent que les systèmes de transport du phosphore dans l'intestin et dans le rein sont, au moins partiellement, différents.

\section{Métabolisme rénal de la 25-OH-D}

De nombreux arguments permettent de penser que le métabolisme rénal de la 25-hydroxy-vitamine $\mathrm{D}_{3}$ $\left(25-\mathrm{OH}-\mathrm{D}_{3}\right)$ est également anormal, tant chez l'homme avec HLX que chez la souris $H y p$. En effet, la concentration plasmatique de $1,25-$ hydroxy-vitamine $\quad \mathrm{D}_{3} \quad$ ( $\mathrm{I} \alpha$, $\left.25-(\mathrm{OH})_{2} \mathrm{D}_{3}\right)$, forme active de la vitamine D, n'est élevée ni chez l'homme porteur de ce syndrome, ni chez la souris $H y p$, en dépit d'une hypophosphorémie significative dont on sait qu'elle stimule la production rénale de I $\alpha, 25-(\mathrm{OH}){ }_{2} \mathrm{D}_{3}$. Nous disposons de preuves plus directes encore d'un métabolisme rénal anormal de la $25-\mathrm{OH}-\mathrm{D}_{3}$ chez la souris $H y p$. En effet, il a été démontré que la production de 24,25-dihydroxy-vitamine $\mathrm{D}_{3} \quad(24$, $\left.25-(\mathrm{OH})_{2} \mathrm{D}_{3}\right)$ par une préparation de mitochondries rénales isolées est plus élevée chez les souris $H y p$ que chez des souris témoins [II] alors que la synthèse de I $\alpha, 25-(\mathrm{OH})_{2} \mathrm{D}_{3}$ est plus basse que celle retrouvée chez la souris normale présentant une hypophosphorémie comparable, obtenue au moyen d'un régime pauvre en phosphore [12]. Il a été montré dans notre laboratoire que la souris $H y p$ présentait une activation anormalement basse de la $25-\mathrm{OH}-$ $\mathrm{D}_{3}$-I-hydroxylase rénale ( $\mathrm{I}-\mathrm{OHase}$ ) après administration de vitamine $\mathrm{D}$ ou de régimes déficients en calcium et que cette réduction de synthèse de I $\alpha, 25(\mathrm{OH})_{2} \mathrm{D}_{3}$ chez la souche mutante était associée à une diminution de la Vmax apparente de l'hydroxylation par l'enzyme mitochondriale [I3, I4] (figure 2). Enfin, des cellules épithéliales rénales en culture provenant de souris $H y p$ ont une réponse anormale de l'activité I-OHase à l'administration de calcitonine ou à la réduction de la concentration de calcium dans le milieu de culture [ ${ }_{5}$ ]

$\mathrm{La}$ synthèse rénale de $\mathrm{I} \alpha$, $25-(\mathrm{OH})_{2} \mathrm{D}_{3}$ est localisée dans le

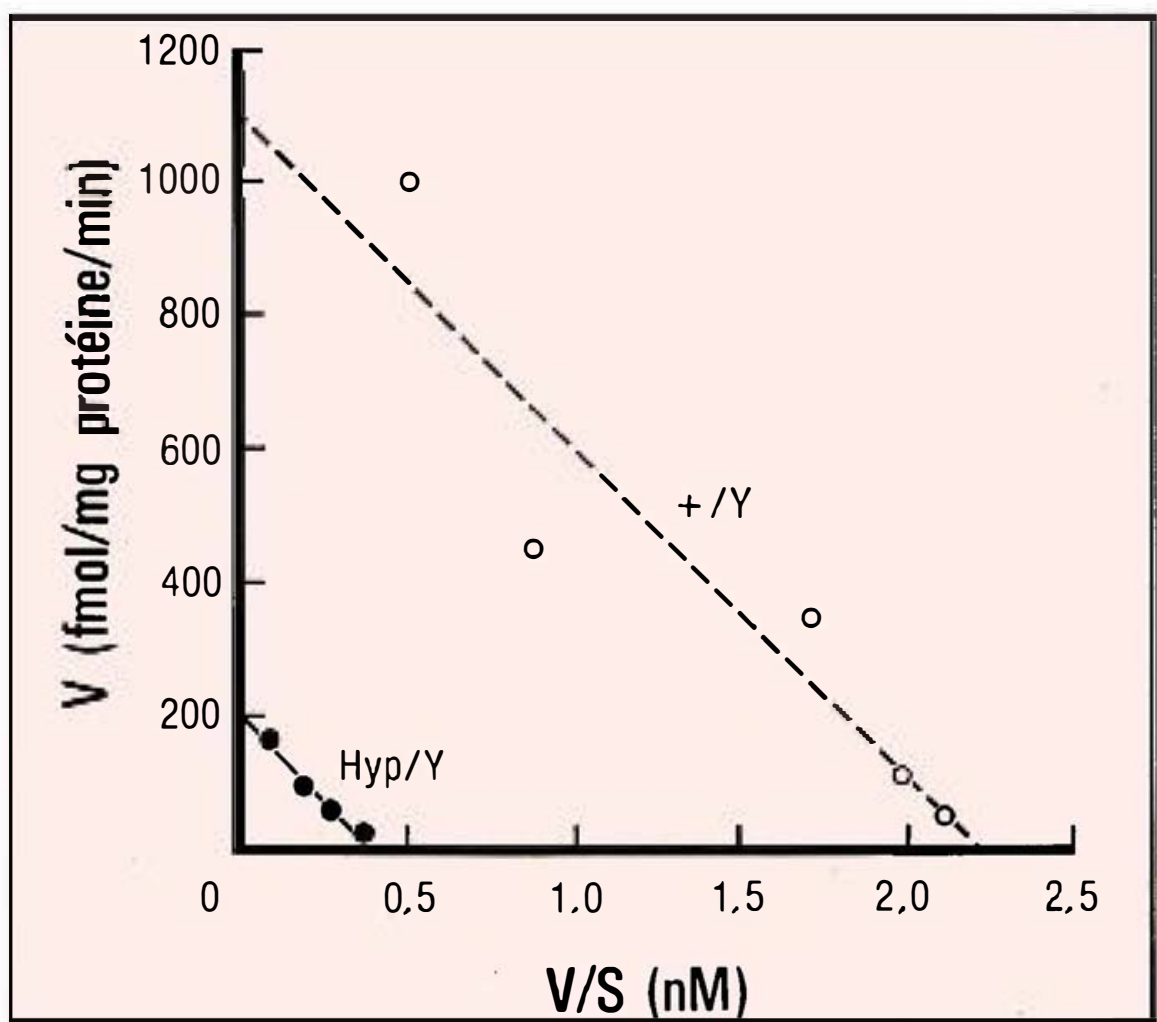

Figure 2. Effets de la concentration de 25-OH-D sur l'activité de la 1-hydroxylase dans des mitochondries rénales de souris normales (O... O) et mutantes (O...) nourries avec un régime carencé en calcium et en vitamine $\mathrm{D}$. $\mathrm{La} 25-\mathrm{OH}-\mathrm{D}_{3}$ tritiée est ajoutée en doses traceuses avec les quantités requises de vitamine $D$ froide (dissoutes dans l'éthanol 95\% dans les milieux d'incubation). L'éthanol est évaporé sous jet d'azote avant l'ajout de la suspension mitochondriale préalablement oxygénée et équilibrée à la température désirée. La figure présente une transformation de Eadie-Hofstee d'une expérience typique présentant les données d'une souris normale (Vmax $=1,096 \mathrm{pmol} / \mathrm{mg}$ protéine $/ \mathrm{min} ; \mathrm{Km}=0,50 \mu \mathrm{M})$ et mutante $(V \max =0,193 \mathrm{pmol} / \mathrm{mg}$ protéine/min; $\mathrm{Km}=0,50 \mu \mathrm{M})$. Les valeurs moyennes des paramètres cinétiques ont été calculées à partir de cinq expériences pour chaque génotype. Valeurs moyennes: Vmax $=1,06 \pm 0,12$ et $0,21 \pm 0,02 \mathrm{pmol} / \mathrm{mg}$ protéine/min pour les souris normales et mutantes respectivement. $K m=0,50 \pm 0,08$ et $0,55 \pm 0,05 \mu \mathrm{M}$ pour les souris normales et mutantes respectivement. 


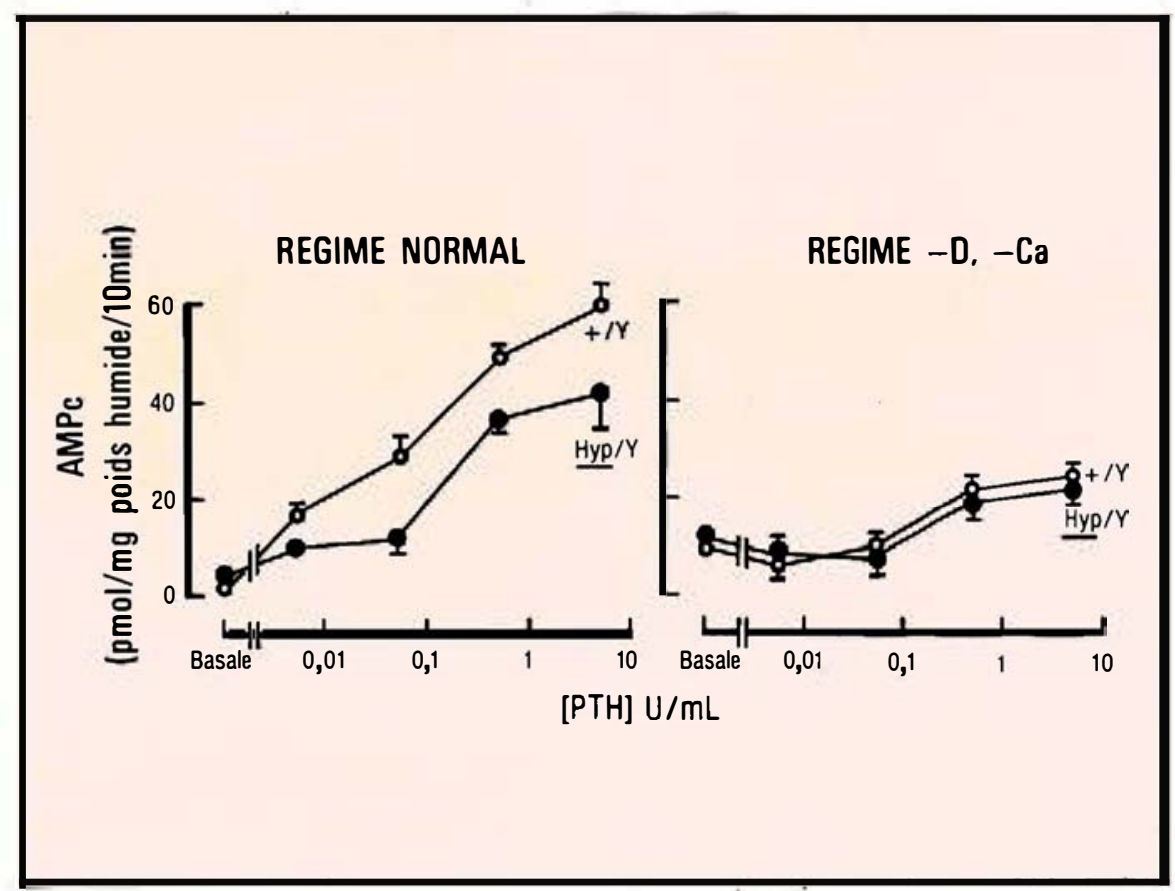

Figure 3. Effets combinés d'un régime carencé en calcium et en vitamine $D$ sur l'accumulation d'AMPC induite par la PTH dans des tranches de cortex rénal de souris normales (O) ou mutantes (O). Les tranches sont incubées à $37^{\circ} \mathrm{C}$ en présence de concentrations croissantes de PTH. Chaque point représente la moyenne \pm un écart-type des déterminations faites en double au cours de quatre expériences distinctes. Le contenu de base en AMPc n'a pas été soustrait avant l'analyse statistique. Effet du génotype, régime normal : $p<0,005$; effet de la vitamine.$D$ carencée chez les deux génotypes : $p<0,001$.

tube proximal chez le poulet et le rat. Il est par conséquent probable que ce soit là aussi que se produise le défaut de métabolisme de la vitamine D chez la souris $H y p$, le tube proximal étant également le siège de l'anomalie de transport du phosphore chez la souris mutante [4].

\section{Hypothèses}

Les relations entre les anomalies de transport du phosphore et le métabolisme de la vitamine $\mathrm{D}$ chez la souris $H y p$ ne sont pas clairement comprises. En effet, dans les désordres mendéliens, un seul gène mutant est considéré comme responsable de toutes les anomalies biochimiques et cliniques rencontrées. Sur la base des données actuellement disponibles, plusieurs hypothèses peuvent être formulées pour expliquer la nature du défaut primaire chiez la souris $H y p$. la résonance magnétique nucléaire n'ont cependant pas démontré une telle différence dans les concentrations intracellulaires de phosphate inorganique $\left[2, I_{7}\right]$ ou de composés phosphorylés [17]. De plus, l'addition de phosphore in vitro à des mitochondries rénales isolées de souris Hyp ne permet pas de corriger le déficit de I-hydroxylation, suggérant ainsi que la diminution d'activité de la I-hydroxylase dans la souche mutante n'est pas le résultat d'une concentration inadéquate de phosphore dans l'environnement enzymatique [14]. De même, l'élévation de l'activité 24-hydroxylase dans les mitochondries de ces souris n'est pas ramenée à la normale par l'addition de phosphore ni in vivo ni in vitro [ $\mathrm{II}$ ]. Il demeure néanmoins impossible d'exclure l'éventualité qu'une perturbation localisée de la concentration en phosphore inorganique dans une fraction cellulaire spécifique puisse être responsable de certaines anomalies de régulation du métabolisme de la vitamine D dans le tube proximal de la souris $H y p$. En dernière analyse, des études réalisant l'isolement, la purification, la reconstitution et la caractérisation d'un système de protéines responsables du transport membranaire du phosphore dans les bordures en brosse de souris normales et mutantes devraient off rir des informations plus directes sur la validité de cette hypothèse.

Première hypothèse : le produit génétique mutant chez la souris Hyp est une protéine responsable du transport du phosphore qui réside dans la bordure en brosse du tube proximal du rein de cette espèce. Un défaut sélectif de transport du phosphore par la membrane de bordure en brosse pourrait perturber la concentration intracellulaire ou la distribution de l'anion phosphate dans la cellule, et ceci pourrait à son tour altérer la régulation des I-OHase et 24-OHase rénales. En effet, Sabina et collaborateurs [I6] ont rapporté la présence d'une concentration réduite de ribonucléosides triphosphates (ATP) chez la souris $H y p$ et ont suggéré que cette réduction reflétait un apport limité de phosphate à la cellule rénale chez la souris mutante. D'autres groupes qui utilisèrent soit des techniques biochimiques conventionnelles soit
Récemment, une nouvelle maladie, cette fois autosomique récessive, a été décrite : le rachitisme héréditaire hypophosphatémique avec hypercalciurie $(\mathrm{RHHH})^{*} \quad$ [I8]. Contrairement aux patients avec HLX, les porteurs de ce syndrome répondent par une élévation appropriée de leur niveau plasmatique de I $\alpha, 25-(\mathrm{OH})_{2} \mathrm{D}_{3}$ à leur hypophosphorémie, qui résulte aussi d'un

* NDLR. Cette maladie, parfaitement analysée sur le plan génétique, physiologique et thérapeutique par Tieder et coll. [I8] semble en fait avoir été décrite en 1962 par Gentil et coll. [a] et en 1964 par Dent et Friedman [b].

a. Gentil CL, Habib R, Le Tan Vinh, et al. Nanisme avec rachitisme, hypercalciurie et protéinurie (deux observations). Ann Pedriatr (Paris) I962; 9: $164-72$.

b. Dent CE, Friedman M. Hypercalciuric rickets associated with renal tubular damage. Arch Dis Child I964; 39: 240-9. 
défaut "primaire " de réabsorption rénale du phosphate [18]. Ces. patients peuvent être traités de façon efficace par le phosphore seul, contrairement aux patients HLX pour lesquels phosphore et vitamine $\mathrm{D}$, en combinaison, sont nécessaires [I]. Ces observations démontrent donc que les lésions génétiques et biochimiques responsables des anomalies des patients RHHH et HLX sont différentes. S'il demeure possible que les patients RHHH et HLX aient une mutation primaire similaire impliquant le transport membranaire de phosphore, le défaut chez ces derniers ainsi que chez la souris $H y p$ doit être plus complexe.

Deuxième hypothèse : l'anomalie génétique chez la souris Hyp implique les systèmes de $\mathrm{I}$ ou de 24-hydroxylation atteignant le métabolisme de la $25-\mathrm{OH}-\mathrm{D}_{3}$. Cette hypothèse repose sur la démonstration que la I $\alpha, 25-(\mathrm{OH})_{2}$ $\mathrm{D}_{3}$ stimule le transport du phosphore par des cellules rénales en culture [19] ainsi que dans des vésicules de bordure en brosse d'animaux préalablement traités avec cette hormone [20]. On pourrait donc penser qu'une production inadéquate de la forme active de la vitamine $\mathrm{D}$ puisse compromettre la réabsorption rénale de phosphore. Cependant l'administration de doses supraphysiologiques de I $\alpha$, 25- $(\mathrm{OH})_{2} \quad \mathrm{D}_{3}$ à des souris $H y p$ ne corrige pas le défaut de transport du phosphore retrouvé sur les bordures en brosse de ces animaux [21]. De même, le traitement des patients porteurs de HLX avec de la $\mathrm{I} \alpha$, ${ }_{25}-(\mathrm{OH})_{2} \quad \mathrm{D}_{3}$ ne corrige pas non plus la fuite rénale en phosphore [I]. Notons cependant que l'homéostasie en phosphate est améliorée avec ce traitement chez les souris $H y p$ comme chez les patients HLX par suite de l'accroissement du transport intestinal du phosphate produit par cette manœuvre [I, 2 I] En dépit des résultats présentés plus haut, cette hypothèse ne peut pas être éliminée tant qu'une meilleure compréhension des bases moléculaires de la régulation de la I- et de la 24-hydroxylation mitochondriale de la vitamine $\mathrm{D}$ n'est pas obtenue et que des compa$m / s n^{\circ} 8$, vol. 2 , octobre 86

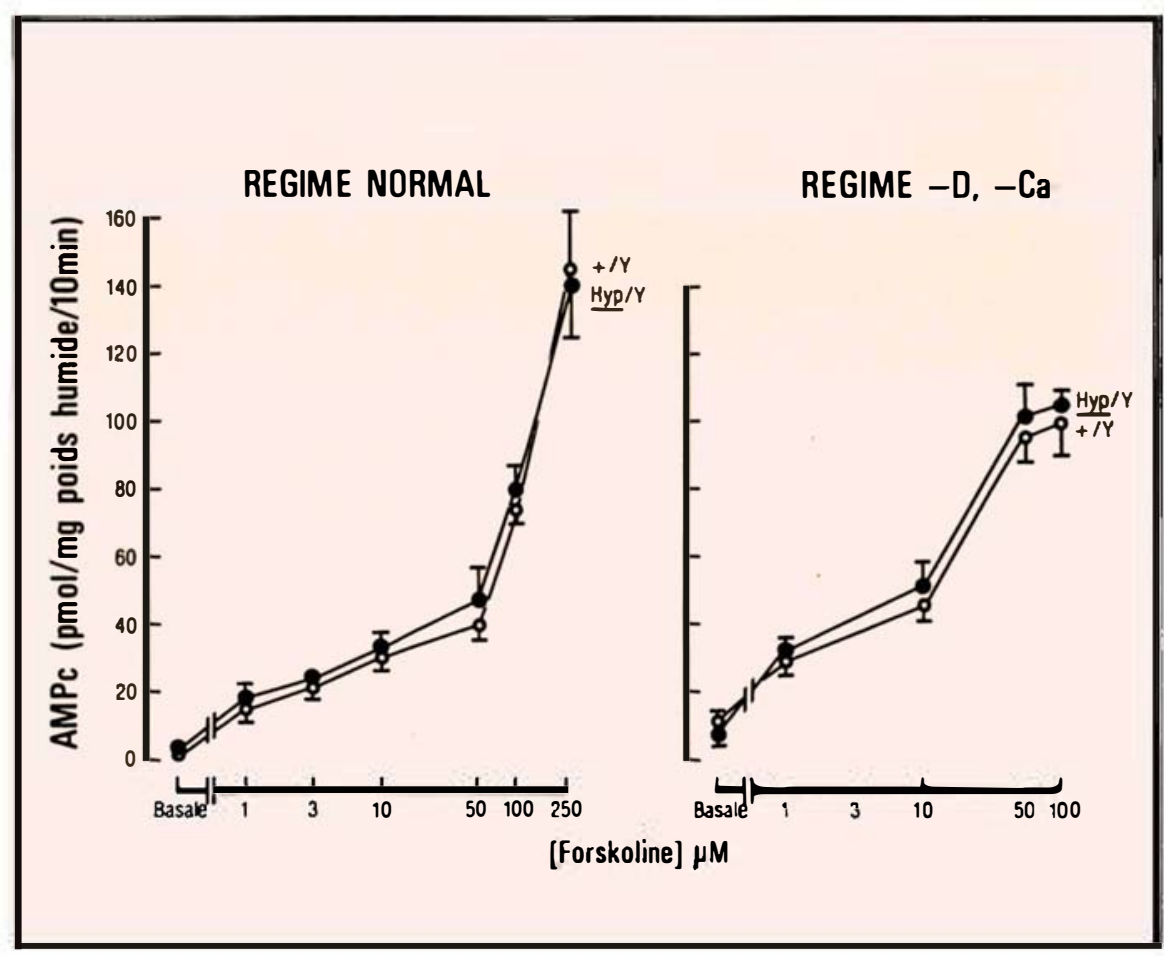

Figure 4. Effets combinés d'un régime carencé en calcium et en vitamine $D$ sur l'accumulation $d^{\prime} A M P c$ induite par la forskoline dans des tranches de cortex rénal de souris normales (O) ou mutantes (O). Les commentaires de la figure 3 s'appliquent à cette figure.

raisons directes entre les systèmes enzymatiques impliqués chez les souris normales et les souris $H y p$ ne sont pas établies.

Troisième hypothèse : Le produit génétique mutant chez la souris Hyp implique un système de régulation enzymatique qui peut modifier à la fois le transport rénal du phosphore et le métabolisme rénal de la vitamine $D$. A l'appui de cette hypothèse, il faut relever le fait que le transport rénal de phosphore [22] comme la synthèse de vitamine $\mathrm{D}[23]$ sont tous deux influencés par des hormones et l'environnement métabolique mettant en jeu l'hormone parathyroïdienne (PTH), l'hormone de croissance, la calcitonine, l'acidose métabolique et les manipulations diététiques du phosphore.

Réponse rénale à la PTH. La PTH est un régulateur important du transport du phosphore par les bordures en brosse du tube proxi- mal [22] ainsi que de l'activité mitochondriale de I-hydroxylation de la vitamine D [23]. Il est par conséquent important d'établir si la réponse rénale à la $\mathrm{PTH}$ est normale ou non chez la souris $H y p$. Brunette et coll. ont rapporté que la production d'AMP cyclique secondaire à une stimulation par la PTH est significativement plus basse dans des tubes proximaux isolés de souris Hyp que de souris normales [24]. Une réponse moindre à la $\mathrm{PTH}$ du système adénylate cyclase a également été démontrée sur des tranches rénales de souris $H y p$ [25] ( $f$ gure 3). Cependant, le fait que la stimulation de l'adénvlate cyclase par la forskoline ${ }^{*}$ soit normale dans le rein des souris mutantes [25] (figure 4) indique que la réduction de synthèse d'AMP cyclique par la

\footnotetext{
- La forskoline est une toxine qui stimule directement ladénylate cyclase et donc augmente la concentration intracellulaire en $A M P$ cyclique, sans passer par I'intermédiaire d'un récepteur hormonal.
} 


\section{REEERENCES}

16. Sabina RL, Drezner MK, Holmes EW. Reduced renal cortical ribonucleoside triphosphate pools in three different hypophosphatemic animal models. Biochem Biophys Res Commun 1982; 109: 649-55.

17. Brown CE, Wilkie CA, Meyer MH, Meyer RA. Response of tissue phosphate content to acute dietary phosphate deprivation in the X-linked hypophosphatemic mouse. Calcif Tiss Int 1985; 37: 423-30.

18. Tieder M, Modai D, Samuel R, et al. Hercditary hypophosphatemic rickets with hypercalciuria. $N$ Engl 7 Med 1985; 312: 61 1-7.

19. Liang CT, Barnes J. Cheng L, Balakir R Sacktor B. Effects of $1,25-(\mathrm{OH})_{2} \mathrm{D}_{3}$ administered in vivo on phosphate uptake by isolated chick renal cells. Am 7 Physiol 1982; 242: C 312-8.

20. Kurnik BR, Hruska KA. Effects of 1,25 dihydroxycholecalciferol on phosphate transport in vitamin D-deprived rats. Am 7 Physiol 1984; 244: F $177-82$.

21. Tenenhouse HS, Scriver CR. Effect of 1,25 dihydroxyvitamin $D_{3}$ on phosphate homeostasis in the X-linked hypophosphatemic ( $H y p)$ mouse Endocrinology 1981; 109: 658-60.

22. Lang F, Greger R, Knox FG, Oberleithner $\mathrm{H}$. Factors modulating the renal handling of phosphate. Renal Physiol I981; 4: I-I6.

23. Fraser D. Regulation of the metabolism of vitamin D. Physiol Rev 1980; 60: 551-613.

24. Brunette MG, Chabardes D, Imbert-Tebou M, Clique A, Montegut M, Morel F. Hormonesensitive adenylate cyclase along the nephron of genetically hypophosphatemic mice. Kidney Int I 979; I 5: 357-69.

25. Tenenhouse HS, Veksler A. Effect of the $H y p$ mutation and diet-induced hyperparathyroidism on renal PTH and forskolin-stimulated cAMP production and brush border membrane phosphate transport. Endocrinology 1986; 118 : $1047-$ 53.

26. Noland TA, Henry HL. Protein phosphorylation in chick kidney. Response to parathyroid hormone, cAMP, calcium, and phosphatidy serine. 7 Biol Chem 1983; 258: 538-46.

27. Hammerman MR, Chase LR. Pi Transport, phosphorylation, and dephosphorylation in renal membranes from $H y p / Y$ mice. Am 7 Physiol 1983; 245: F 701-6.

28. Tenenhouse HS, Henry HL. Protein kinase activity and protcin kinase inhibitor in mousc kidney: Effect of the X-linked $H y p$ mutation and vitamin D status. Endocrinology 1985; 61: 171926.

29. Nishizuka Y. Protein kinases in signal transduction. Tibs 1984; 9: 163-6.

30. Barrett PQ Zawalich K, Rasmussen H. Protein kinase $C$ activity in renal microvillus membranes. Biochem Biophys Res Commun 1985;

PTH ne peut pas être attribuée à une activité catalytique anormale de l'adénylate cyclase, mais suggère plutôt un couplage anormal entre les récepteurs de la PTH et le système adénylate cyclase. Un tel mécanisme a été invoqué récemment pour expliquer la résistance à la PTH créée par l'hypophosphatémie chez le chien.

Nous avons démontré dans notre laboratoire que les souris $H y p$ présentaient une augmentation appropriée de la PTH circulante à la suite d'une carence nutritionnelle en vitamine $D$ et en calcium [14]. De plus, l'hyperparathyroïdie induite par cette manipulation diététique est associée chez la souris mutante à une augmentation appropriée de l'excrétion d'AMP cyclique urinaire et de l'excrétion fractionnelle des phosphates [14]. De plus, une carence en calcium ou en vitamine $\mathrm{D}$ réduit le transport de phos- phore couplé au sodium chez les deux types de souris alors que le transport de glucose demeure inchangé [25] (figure I). Dans ce contexte, la persistance d'un défaut membranaire de transport chez la souris $H y p$ indique que l'expression du phénotype rénal est indépendante de la PTH chez ces animaux [25] (figure I). Une déficience en vitamine $D$ et en calcium atténue de façon significative la production d'AMP cyclique sous influence de la PTH chez les souris normales comme chez les souris $H y p$; dans ces conditions, les différences génotypiques de l'activité adénylatecyclase ne sont donc plus apparentes [25] (figure 3). Au contraire, la stimulation de la synthèse d'AMP cyclique par la forskoline n'est pas modifiée par des changements diététiques [25] (figure 4). Ces résultats sont donc en accord avec l'existence d'un défaut de couplage

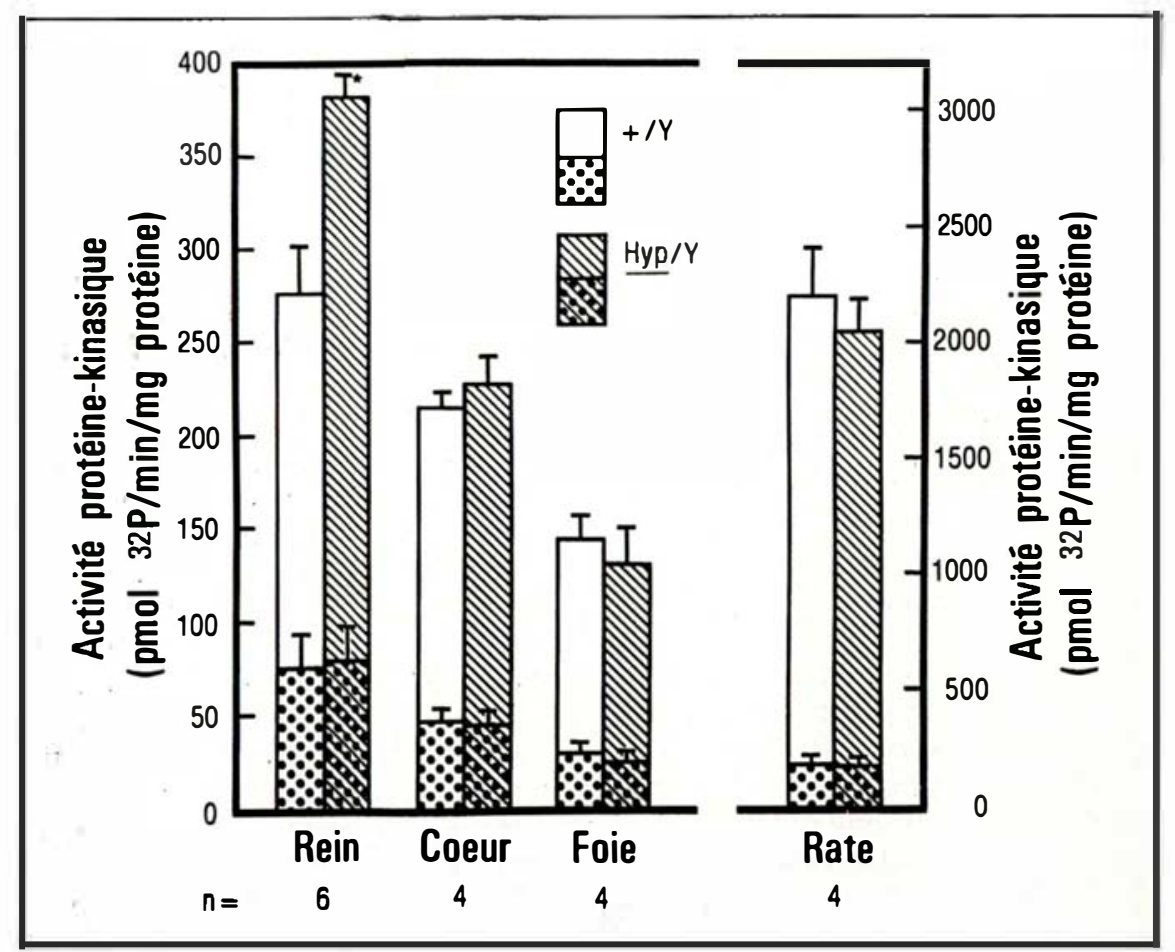

Figure 5. Vitesse initiale de l'activité de la protéine kinase $C$ au niveau du surnageant $20000 \mathrm{~g}$ préparé à partir de rein, de cœur, de foie et de rate de souris normales $(+/ Y)$ et/ou mutantes $(H y p / Y)$. Les zones pointillées représentent l'activité de la protéine kinase mesurée en l'absence de calcium, de phosphatidy/sérine et de dioléine. Les écarts-types de la moyenne sont calculés à partir du nombre de souris examinées séparément dans chaque groupe.

* La protéine kinase $C$ est élevée de façon significative chez les Hyp par rapport aux souris $+/ Y(p<0,001)$ 
entre les récepteurs à la PTH et le système adénylate-cyclase induit par le régime chez les souris normales, aussi bien que chez les mutants $H y p$. Ce mécanisme ne semble donc pas être à l'origine des désordres observés.

Les protéines kinases dans le rein. Les effets de la PTH sur le rein sont en général attribués à une activation de protéines kinases cytoplasmiques et membranaires sous l'effet de l'accumulation cellulaire d'AMP cyclique [26]. Les protéines kinases activées vont à leur tour stimuler la phosphorylation de protéines endogènes spécifiques. Cette phosphorylation induit un changement dans l'activité de ces protéines qui serait responsable de l'expression physiologique finale de la PTH.

I 'effet de la mutation $H y p$ sur la phosphorylation des protéin $: s$ de bordure en brosse par des protéines kinases endogènes (associées aux bordures en brosse) a été étudié par deux laboratoires. Les résultats de ces études ne révèlent aucune différence génotypique dans le type électrophorétique des composés phosphorylés dans les conditions basales ou après stimulation par l'AMP cyclique. De plus, le rythme de déphosphorylation d'une protéine de poids moléculaire 62000 , préalablement phosphoryléc sous l'influence de l'AMP cyclique, est identique chez la souris normale et chez la souris $H y p$, éliminant l'hypothèse selon laquelle un défaut dans les phosphatases associées aux bordures en brosse expliquerait la persistance d'un transport de phosphore réduit après parathyroïdectomie chez la souris $H y p$ [27].

Afin d'essayer d'expliquer les mécanismes reliant le défaut rénal de transport du phosphore et celui du métabolisme de la vitamine $\mathrm{D}$ chez la souris mutante, Tenenhouse et Henry ont examiné une protéine kinase cytosolique rénale, ainsi qu'un inhibiteur protéique de cette protéine kinase, chez les souris normales et les souris mutantes [28]. Leurs résultats démontrent que la protéine kinase dépendante de l'AMP cyclique et la protéine inhibitrice de cette activité sont similaires dans les deux génotypes.
Cependant, l'activité d'une protéine kinase stimulée par le calcium et les phospholipides (protéine kinase C) est significativement plus élevée dans le cytosol rénal de la souris Hyp [28] (figure 5). Cette activation de la protéine kinase $C$ n'apparaît pas dans le surnageant d'homogénats de cœur, de rate ou de foie de souris Hyp [28] (figure 5). Ces données suggèrent donc qu'une activité anormalement élevée de protéines kinases $\mathrm{C}$ spécifiquement retrouvée dans le rein des souris $H y p$ puisse être en relation avec le déf aut rénal de transport de la bordure en brosse ainsi qu'avec le défaut de métabolisme mitochondrial de la vitamine $D$.

La protéine kinase $C$ représente un nouveau système de phosphorylation protéique qui est largement répandu chez les eukaryotes, et qui est activé par le calcium, la phosphatidylsérine et le diacylglycérol [20]. Ce système n'est pas affecté par la calmoduline et diffère des protéines kinases activées par les nucléotides cycliques. Il a été proposé que dans des conditions physiologiques, la protéine kinase C est activée par le diacylglycérol qui est formé de façon transitoire dans les membranes, à partir du phosphatidyl-inositol, lorsque les cellules sont stimulées par un ou par une variété de messagers extracellulaires [29]. La protéine kinase $\mathrm{C}$ a été récemment impliquée dans la régulation du transport des électrolytes par l'intestin, et dans la stéroïdogenèse dans les cellules testiculaires. Elle est capable de phosphoryler des protéines membranaires et cytoplasmiques dans le rein du poulet [26] et elle a été récemment identifiée dans des bordures en brosse purifiées de rein de lapin [30].

Il faudra encore beaucoup de travail avant d'élucider le rôle précis de la protéine kinase $C$ dans le rein du mammifère et d'identifier les protéines endogènes phosphorylées par ce système. On peut imaginer cependant que des altérations de la phosphorylation de composants spécifiques de la bordure en brosse et de protéines mitochondriales par le système protéine kinase $C$ puissent être responsables du phénotype rénal chez la souris hypophosphatémique

\section{Summary}

The Hyp mouse, a murine homologue of X-linked hypophosphatemic rickets in man, is characterized by hypophosphatemia, rickets which is not responsive to physiological doses of vitamin $D$ and a reduced capacity of the kidney to reabsorb filtered phosphate. Studies from this laboratory have demonstrated that $H y p$ mice have specific renal defects in brush border membrane phosphate transport and mitochondrial 25-hydroxyvitamin $\mathrm{D}$ metabolism. The precise relationship between these two proximal tubular abnormalities is poorly understood. As in all Mendelian disorders, one mutant gene must be responsible for the biochemical and clinical phenotype. In the present review, several hypotheses are put forth to explain the nature of the primary mutation in the $H_{y^{\prime}} p$ mouse.

\section{TIRES A PART}

H. S. Tenenhouse : Groupe de recherche génétique, institut de recherches, hôpital de Montrćal pour les enfants et département de biologic, centre de génćtique humaine, universitć McGill 2300 ruc Tupper, $\mathrm{H}_{3} \mathrm{H}_{1} \mathrm{P}_{3}$, Montrćal. 